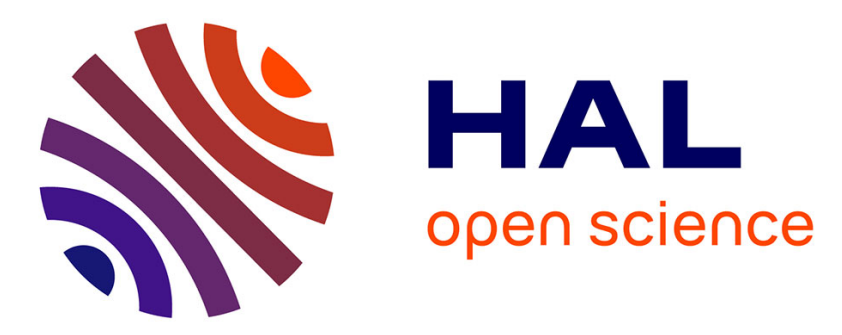

\title{
Effect of surface pattern on the adhesive friction of elastomers
}

Fanny Wu-Bavouzet, Juliette Cayer-Barrioz, Alain Le Bot, Françoise Brochard-Wyart, Axel Buguin

\section{To cite this version:}

Fanny Wu-Bavouzet, Juliette Cayer-Barrioz, Alain Le Bot, Françoise Brochard-Wyart, Axel Buguin. Effect of surface pattern on the adhesive friction of elastomers. Physical Review E : Statistical, Nonlinear, and Soft Matter Physics, 2010, 82, 10.1103/physreve.82.031806 . hal-03127968

\section{HAL Id: hal-03127968 \\ https://hal.science/hal-03127968}

Submitted on 1 Feb 2021

HAL is a multi-disciplinary open access archive for the deposit and dissemination of scientific research documents, whether they are published or not. The documents may come from teaching and research institutions in France or abroad, or from public or private research centers.
L'archive ouverte pluridisciplinaire HAL, est destinée au dépôt et à la diffusion de documents scientifiques de niveau recherche, publiés ou non, émanant des établissements d'enseignement et de recherche français ou étrangers, des laboratoires publics ou privés. 


\title{
Effect of surface pattern on the adhesive friction of elastomers
}

\author{
Fanny Wu-Bavouzet \\ Laboratoire Physico-Chimie Curie, CNRS UMR 168, Institut Curie Section Recherche, Paris, France
}

Juliette Cayer-Barrioz and Alain Le Bot

LTDS, UMR 5513, Ecully, France

Françoise Brochard-Wyart

Laboratoire Physico-Chimie Curie, CNRS UMR 168, Institut Curie Section Recherche, Paris, France

Axel Buguin

Laboratoire Physico-Chimie Curie, CNRS UMR 168, Institut Curie Section Recherche, Paris, France

(Received 7 March 2010; published 29 September 2010)

\begin{abstract}
We present experimental results for the friction of a flat surface against a hexagonally patterned surface, both being made of PolyDiMethylSiloxane. We simultaneously measure forces of range $10 \mathrm{mN}$ and observe the contact under sliding velocities of about $100 \mu \mathrm{m} / \mathrm{s}$. We observe adhesive friction on three different pattern heights $(80,310$, and $2100 \mathrm{~nm})$. Two kinds of contacts have been observed: the flat surface is in close contact with the patterned one (called intimate contact, observed for $80 \mathrm{~nm}$ ) or only suspended on the tops on the asperities (called laid contact, observed for $2100 \mathrm{~nm}$ ). In the range of velocities used, the contact during friction is similar to the static one. Furthermore, our experimental system presents a contact transition during friction for $h=310 \mathrm{~nm}$.
\end{abstract}

DOI: 10.1103/PhysRevE.82.031806

PACS number(s): 61.41.+e, 62.20.Qp

\section{INTRODUCTION}

The development of microfabrication techniques [1-4] has allowed scientists to build microstructured surfaces with asperities of controlled size. Their use in microfluidics and biophysics has led to numerous applications such as reducing friction at an interface liquid/channel [5], mimicking biological system properties such as the hydrophobicity of a lotus leaf [6], or understanding gecko setae outstanding adhesive properties $[7,8]$.

Two types of surfaces can be distinguished: those with a random roughness, and others with a regular pattern. These surfaces have inspired works on contact mechanics (see e.g., $[9,10]$ for a review [11]), adhesion, and friction. In the domain of adhesion, Fuller and Tabor [12] have proposed a force-displacement relation for the contact of flat elastic spheres on a rough substrate whose asperities follow a Gaussian distribution. This relation is based on the application of Johnson-Kendall-Roberts (JKR) theory [13] for the contact between one single asperity and an elastic sphere. An expression of the pull-off force for the separation of both surfaces can be deduced from this relation, in good agreement with experimental data. Persson et al. [14] have studied the contact adhesion when hard roughness occurs on many different length scales, and have introduced the fractal dimension $D_{f}$. This model has pointed out the existence of two types of contacts, one on small roughness $\left(D_{f}<2.5\right)$ where real and nominal area are equal, the other on high asperities for which real contact area is much smaller than the apparent one. In the latter case, the adhesion force may be strongly reduced. More precisely, experimental results on adhesion energy calculations [15] show that the pull-off force expression given by Johnson-Kendall-Roberts theory [13] for an elastic ball in contact with a flat hard substrate is still valid for a rough one: $F_{\text {pull-off }}=\frac{3 \pi}{2} R \Delta \gamma$ where $R$ is the ball radius and $\Delta \gamma$ the interfacial binding energy per unit surface area between two flat surfaces. However, in the case of a rough surface, $\Delta \gamma$ must be replaced by the effective interfacial energy $\gamma_{\text {eff }}$ calculated from the surface roughness power spectra. Other effects such as substrate softness can influence the adhesion. Surface force apparatus (SFA) experiments have shown that stiffness and viscoelasticity of rough surfaces can cause the effective adhesion force to be orders of magnitude lower or higher than the thermodynamic value [16]. Indeed, with soft surfaces as polymers, molecular interdiffusion and bulk deformation can result in high adhesion. Very recently, Verneuil et al. [17] have measured energy adhesion for different heights of pillars and interpreted the results using JKR theory.

In the domain of friction, influence of sliding velocity and normal load on friction is also two important subjects of studies $[18,19]$. Works on sliding velocity influence have led to various models since Schallamach's interpretation of Grosch's works on friction of rubbers against hard flat and rough surfaces. Grosch [19] has found a Williams-LandelFerry mastercurve when studying friction force for various velocities $U$ and temperatures $T(100 \mu \mathrm{m} / \mathrm{s}<U<10 \mathrm{~m} / \mathrm{s}$, and $-60{ }^{\circ} \mathrm{C}<T<90{ }^{\circ} \mathrm{C}$ ). This curve presents one peak on smooth surfaces, due to the adhesive contribution of bindings between rubber and substrate, and another one on rough substrates, due to the rubber bulk deformation [18]. In Schallamach's model, the transition probability of adhesive bonds formation and breaking explaining the adhesive peak is thermally controlled (when not due to an external force), whereas in Chernyak and Leonov's model [20], it is a stochastic process. However, even on a flat surface, viscoelastic losses arise during friction processes, as suggested by Savkoor [21] and Ludema and Tabor [22] (see, e.g., [23] for a 
review). The existence of a peak, and hence of different monotonicities show several regimes in the velocity-friction force curves. At low sliding velocities, a velocity logarithmic increase in friction force has been experimentally observed for the friction of atactic polystyrene (PS) above the glass transition temperature [24], which is consistent with an activated molecular relaxation process. These results have led to the determination of the activation energy. For friction experiments performed on a surface force apparatus (SFA) between two mica surfaces lubricated by a surfactant, Drummond et al. [25] have adapted Chernyak and Leonov's adhesive friction model taking in account the elastic energy stored in an adhesive junction. The results give values for microscopic parameters as the adhesive junction surface. Cayer-Barrioz et al. [26] have investigated the friction of polymers adsorbed on two metallic surfaces on a molecular tribometer derived from a SFA. They have used the same model associated to experimental measurements of shear elastic modulus and interface thickness of the adsorbed layers to deduce the dynamics of the interpenetration zone with sliding velocity. Using the theory on polymers of De Gennes [27] they have correlated the frictional rheology of the interface to the molecular organization of the confined polymeric interface on the surfaces.

In addition, Schallamach [28] studied the normal load dependence $F_{\mathrm{n}}$ of friction coefficient $\mu$ and found $\mu \sim F_{\mathrm{n}}^{-1 / 3}$ for the friction of vulcanized rubbers on a rough plate, which corresponds to the case of a contact area following Hertzian laws. Experiments with regular patterns of polydimethylsiloxane (PDMS) against glass [29] and steel [30] have led to the same conclusions. For all cases described above, the normal load $F_{\mathrm{n}}$ was larger than the adhesive one $\sim W R$, with $W$ the adhesive energy and $R$ the rubber block characteristic size, which explains that the contact follows the non adhesive limit of Johnson-Kendall-Roberts' laws. Other authors [31] have studied the corresponding intensive lengths and found that tangential stress $\tau$ is a linear function of pressure $P$ in a limited range of pressure values $P\left(10^{8} \mathrm{~Pa}<P\right.$ $\left.<10^{9} \mathrm{~Pa}\right): \tau=\tau_{0}+\alpha P$ where $\tau_{0}$ and $\alpha$ are two constants. $\tau$ is the shear yield stress of the asperities. Homola et al. [32] has introduced two parameters $S_{\mathrm{c}}$ and $\alpha: F_{\mathrm{t}}=A S_{\mathrm{c}}+\alpha F_{\mathrm{n}}$ with $A$ the real contact area. This can be seen as a generalization of Coulomb law with $\mu=\alpha . S_{\mathrm{c}}$ is the shear stress determined by intermolecular forces and $\alpha$ the coefficient of friction, which for static friction is simply $\tan \theta$. The latter law has been experimentally observed $[33,34]$. In the case of low adhesion, Coulomb laws are observed $[32,35,36]$. For the friction of PolyMethylMethAcrylate (PMMA) on a silicone wafer recovered by a trimethylsilane (TMS) layer (single contact on a flat substrate), Bureau [37] found a logarithmic dependence of tangential stress as a function of pressure. Persson [38] suggests the influence of capillary waves to explain this result. Except for the works of [37], the case where $\sigma$ depends on $P$ occurs generally for multiasperities contact, where real contact area is smaller than the apparent one. In the case of saturated contacts, no dependence is expected [39].

The effect of roughness on the friction coefficient has been studied by [40]. A flat mica surface slides against a rough PolyUrethane one (elastic modulus about $1 \mathrm{GPa}$ ). The
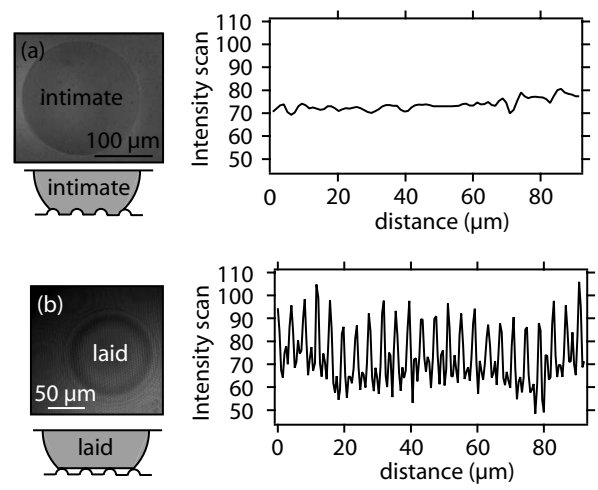

FIG. 1. (a) Intimate contact: picture, schematic of the lens in close contact with the substrate, and linear intensity profile inside the contact. (b) Laid contact: picture, schematic of the lens suspended on the asperity tops, and intensity profile inside the contact.

RMS roughness varies from 0.5 to $7.1 \mathrm{~nm}$, while the average radius of the asperity tips is about a few micrometers. The friction is controlled by adhesion, and the decrease in the friction force with increasing roughness can be understood considering the plasticity index of the surfaces and using a theory developed by Kogut and Etsion [41].

Various contact instabilities are also observed at sliding interfaces $[42,43]$ : they occur at different length scales from molecular to macroscopic one [36]. For instance, in 1971, Schallamach [43] discovered that friction of elastomeric interface is often dominated by the onset and propagation of elastic instabilities in the form of surface waves. The latter propagate through the contact from the leading to the trailing edge as soon as the sliding velocity exceeds a threshold value which varies with the normal load and/or the radius of curvature of the slider. To form Schallamach waves, the adhesive force at the interface must be strong enough to pin the interface at the rear of the contact area creating a zone of tension, while shearing the interface causes a zone of compression in the front of the contact area [36]. Another typical example of friction instabilities with elastomer is the occurrence of stick-slip motion or sprag slip phenomenon, succession of stick and slip phases. The common driving mechanism is most often attributed to a negative slope for friction as a function of velocity.

The first goal of this paper is to propose an adhesive friction law for elastomeric interfaces. It derives from an experimental investigation of the sliding velocity and normal load influence on the steady state friction of a PolyDiMethylSiloxane (PDMS) lens on a controlled patterned surface. Three hexagonal networks of pillars of height 80, 310, and 2900 $\mathrm{nm}$ have been used to allow us to define two states of the contact $[17,23]$ :

(i) The intimate contact [23], corresponding to the lowest pillars, where the top lens follows the modulation of the substrate pillars [see Fig. 1(a)]. The contact has an homogeneous appearance, as shown by an intensity scan inside the contact.

(ii) The laid contact, corresponding to the highest pillars where the top lens is laid on top of the substrate pillars [see Fig. 1(b)]. An intensity scan along an asperities line shows the intensity modulations due to the presence of an air film between the lens and the patterned substrate. 


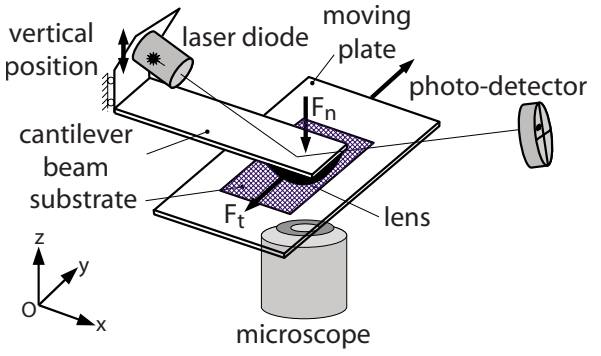

FIG. 2. (Color online) Principle of the experimental setup. A PDMS lens (black) placed at the extremity of a cantilever beam is pressed against a PDMS textured substrate (hachured zone). The contact zone is observed with a microscope. A constant sliding speed $U$ is applied to the substrate. The normal force $F_{n}$ and friction force $F_{t}$ respectively result in a bending and a torsion of the cantilever beam measured by the deviation of a laser beam.

The frictional response of these contacts is studied. In addition, this paper enlightens the coexistence of a mixed contact, i.e., a contact partly intimate and partly laid, and it focuses on its dynamics during friction.

\section{EXPERIMENTAL SYSTEM}

The principle of the experiment is the following. A lens made of PolyDimethylSiloxane (PDMS) is pressed against a patterned PDMS substrate. The latter is a plane surface on which a regular pattern of pillars has been realized. The contact is observed with a microscope placed under the substrate. The substrate is pulled with a constant sliding velocity $U$. The lens is a smooth convex surface placed at the extremity of a flexible cantilever beam. The applied normal load $F_{n}$ and the resulting friction force $F_{t}$ are known during the sliding by measuring with a photodetector the deviation of a laser beam reflected at the beam end, following a principle used in atomic force microscopy (Fig. 2).

The substrate is laid on a glass slide which is driven by two de motors (860A-2, Newport, Irvine, USA). The sliding velocity $U$ ranges from $35 \mu \mathrm{m} / \mathrm{s}$ to $350 \mu \mathrm{m} / \mathrm{s} \pm 10 \%$.

The cantilever beam is a glass plate of dimension $22 \mathrm{~mm} \times 1 \mathrm{~mm} \times 200 \mu \mathrm{m}$. Its extremity is covered with a thin gold layer of thickness $50 \mathrm{~nm}$ to reflect the laser beam. When the lens is pressed against the substrate with a normal load $F_{n}$, the beam bends along $z$-axis. The vertical stiffness $k_{n}$ is defined by $F_{n}=k_{n} \Delta z$ where $\Delta z$ is the vertical deflection of the beam end. It is experimentally determined by measuring the resonance frequency of the beam loaded with different weights [44]. Its value is about $k_{n} \sim 10 \mathrm{~N} / \mathrm{m}$. In other respects, when the substrate slides, a tangential or friction force $F_{t}$ is applied to the top of the lens (contact point). It results in a torque applied to the beam along the $x$ axis. By denoting $\Delta y$ the horizontal displacement of the contact point, the torsion stiffness $k_{t}$ is defined by $F_{t}=k_{t} \Delta y$. It is related to the vertical stiffness by

$$
k_{t}=k_{n} \frac{2}{3(1+\nu)}\left(\frac{l}{e+t / 2}\right)^{2},
$$

where $l$ is the beam length, $t$ the beam thickness and $e$ the lens height. Typical values are $k_{t} \sim 1000$ to $10000 \mathrm{~N} / \mathrm{m}$. The resonance frequencies of the set cantilever beam-lens, have been assessed by closed-form relationships based on EulerBernoulli's beam for flexural motion and classical theory of rod for torsional motion [45]. The first eigenfrequencies are $\sim 100 \mathrm{~Hz}$ for $z$-flexion, $\sim 900 \mathrm{~Hz}$ for $y$-flexion and $\sim 44000 \mathrm{~Hz}$ for torsion. These large values are due to the lightness of the lens. They give the upper frequency limit of the force measurement which is particularly important for transient phenomena.

The laser supplied by a diode is specularly reflected at the extremity of the beam and hints the center of four-quadrant photodetector. When the normal load $F_{n}$ is exerted, the laser is deviated upward along the $z$ axis. When the friction force $F_{t}$ is applied to the lens base, the torsion of the cantilever beam results in a deviation along the $y$ axis of the laser. The tensions $U_{n}$ and $U_{t}$ measured by the photodetector are therefore related to forces $F_{n}$ and $F_{t}$ respectively with $F_{n}$ $=k_{n} a_{n} U_{n}$ and $F_{t}=k_{t} a_{t} U_{t}$. The coefficient $a_{n}$ is experimentally determined by measuring the resulting voltage $U_{n}$ for different values of the vertical deflection $\Delta z$ of the cantilever beam. $\Delta z$ is measured with a micrometer. A similar method is used for the coefficient $a_{t}$.

The vertical position of the set cantilever beam-laser diode-photodetector is controlled with a piezoelectric crystal (NanoX400SG, Piezojena). A servo-control loop allows to maintain the normal load $F_{n}$ constant. The normal load $F_{n}$ ranges from $100 \mu \mathrm{N}$ to $500 \mu \mathrm{N}$ with a resolution of $10 \mu \mathrm{N}$.

A data acquisition card (Keithley KUSB 3116, Cleveland, USA) records the sliding velocity $U$, the piezoelectric voltage and the four-quadrant photodetector output signals $U_{n}$ and $U_{t}$.

Lens and substrate are made of PolyDiMethylSiloxane (PDMS) realized by mixing polymer chains $(N \sim 350)$ from Sylgard 180 (Dow Corning) and curing agent in 10:1 weight ratio. Air bubbles formed during mixing are removed in vacuum bell. The prepolymer is cured for $48 \mathrm{~h}$ at $65^{\circ} \mathrm{C}$ for reticulation. The obtained PDMS has a Young's modulus $E$ $\sim 1 \mathrm{MPa}$ and a Poisson's coefficient $\nu=0.5$ [46].

For the lens, droplets of contact angle between $55^{\circ}$ and $65^{\circ}$ are deposited on a hydrophobic microscope slide and then cured for reticulation. The droplet (lens) and the slide (cantilever beam) are then fixed on the apparatus. The substrate made of the same PDMS, is molded. The mold is a silicium wafer of area $1 \mathrm{~cm}^{2}$ printed by photolithography and reactive ion etching/deep reactive ion etching. The mold is covered with the prepolymer and cured for reticulation. The PDMS layer is then peeled. The surface texture is a hexagonal lattice of cylindrical pillars of diameter $2 \mu \mathrm{m}$. The pillars are separated by a distance of $4 \mu \mathrm{m}$ between two successive centers. Since the lattice is hexagonal, the theoretical occupation rate is $23 \%$. Three different types of substrate are used respectively with pillar height $h=80 \mathrm{~nm}, h$ $=310 \mathrm{~nm}$, and $h=2900 \mathrm{~nm}$ (Fig. 3).

The contact is observed continuously during sliding with an inverted microscope (Axiovert 135, Zeiss, Switzerland) by reflection interference microscopy. The interferences fringes are clearly visible and provide a direct measurement of the contact radius $a$ and the lens profile. Typical size of contact radius ranges from 50 to $100 \mu \mathrm{m}$ and can be measured with a resolution of $1 \mu \mathrm{m}$. 
(a)

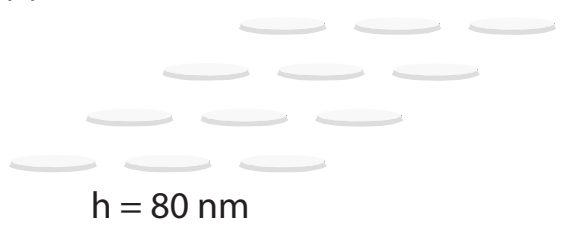

(b)

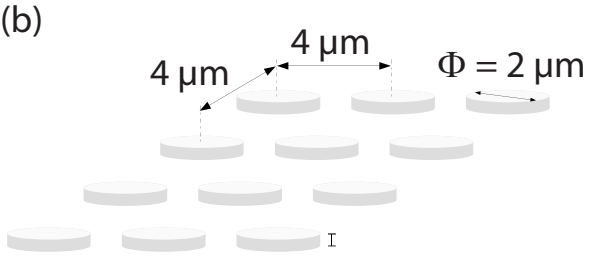

$\mathrm{h}=310 \mathrm{~nm}$

(c)

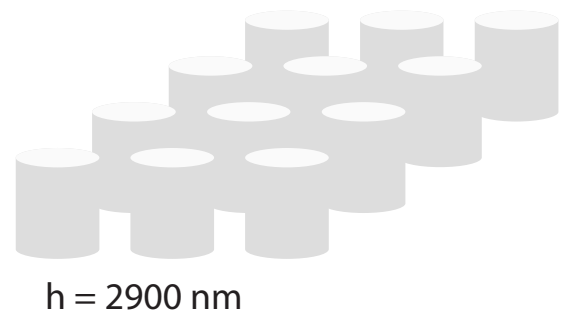

FIG. 3. PDMS substrate texture. A hexagonal pattern of cylindrical pillars (diameter: $2 \mu \mathrm{m}$ ) separated by $4 \mu \mathrm{m}$ is realized on the surface. Three different types of substrate with height (a), $h$ $=80 \mathrm{~nm},(\mathrm{~b}), h=310 \mathrm{~nm}$, and (c), $h=2900 \mathrm{~nm}$ are used.

\section{RESULTS AND DISCUSSION}

\section{A. Static contact}

For a static contact, two types of contact have been observed. For a substrate with small pillars $(h=80 \mathrm{~nm})$, an intimate contact between lens and pillars is observed [see Fig. 1(a)]: the contact area is uniform. On the contrary, on a substrate with high pillars, i.e., $h=2900 \mathrm{~nm}$, we can distinguish the pillars tops inside the contact area [see Fig. 1(b)]: the lens is laid on these pillars tops, and an air film comes in between lens and pillars. These observations can be explained by the competition between the two phenomena governing the contact properties between the lens and the substrate: adhesion and elastic deformation energies [17]. For $h=80 \mathrm{~nm}$, the adhesion energy is much larger than the elastic deformation cost. This allows the lens to follow the pillars modulations and be in intimate contact. Above a critical height $h_{c}$, the elastic energy dominates and the lens is laid only on the pillars tops, without invading the interspace between the pillars.

$h_{c}$ represents the static transition height from a laid contact to an intimate contact at zero compression force. It is given by the balance between adhesion energy on a flat surface $W_{0} a_{1}^{2}$, where $W_{0}$ is the adhesion energy per unit surface on a flat substrate and $a_{1}$ the contact radius between the lens and one single pillar, and the cost due to the lens deformation on a pillar $E h^{2} a_{1}$, with $E$ the Young modulus of the system. As $a_{1}$ reaches $r$ at the transition, we obtain

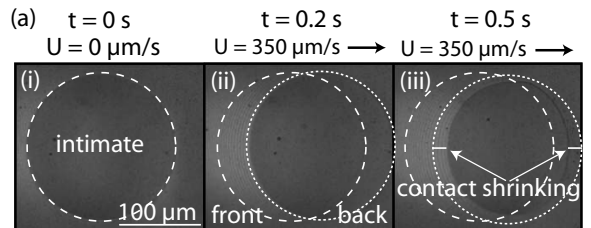

(b)

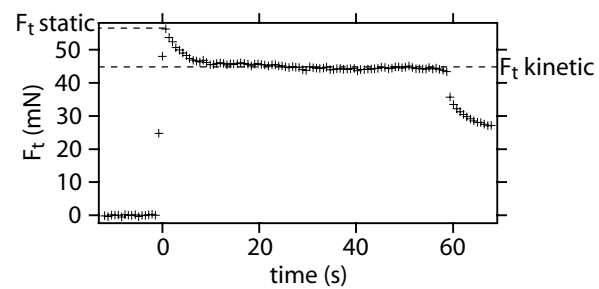

FIG. 4. $h=80 \mathrm{~nm}$ : (a) Contact images when sliding begins: (i) before friction begins, and (ii) and (iii) during friction. The arrow represents the sliding direction of the substrate. (b) Friction force versus time for a constant normal load of $70 \mu \mathrm{N}$ and a sliding velocity of $35 \mu \mathrm{m} / \mathrm{s}$.

$$
h_{c} \sim \sqrt{\frac{W_{0}}{E} r} .
$$

With $W_{0}=43 \mathrm{mN} / \mathrm{m}, E=1.1 \mathrm{MPa}, r=1 \mu \mathrm{m}$ [17] we find $h_{c} \simeq 200 \mathrm{~nm} . h_{c}$ is experimentally found to lie between 220 and $310 \mathrm{~nm}$ and is in good agreement with theoretical predictions [17].

\section{B. Adhesive friction law}

For $h=80 \mathrm{~nm}$, and for the range of velocities and normal forces used during our experiments, we observe that the contact remains entirely intimate during friction. For $h$ $=2900 \mathrm{~nm}$, we observe that it stays entirely laid. For $h$ $=310 \mathrm{~nm}$, we observe the existence of a mixed contact [see Fig. 8ii]. The back of the lens is intimate, whereas the front is laid. This phenomenon is due to the fact that the pillars have a height close to the static laid/intimate transition height $h_{c}$.

Our PDMS/PDMS experimental system [17] presents high adhesion energies. It is thus expected that adhesion plays an important role in friction experiments. Let us estimate the adhesive force, and compare it to the normal load applied: we find that the adhesion force $W \times$ lens radius $\sim 40 \times 10^{-3} \mathrm{mN} / \mathrm{m} \times 10^{-3} \mathrm{~m} \sim 40 \mu \mathrm{N}$ is of same order of magnitude as normal load $F_{n} \sim 100 \mu \mathrm{N}$. Therefore, we propose an adhesive friction law, where the friction force is proportional to the contact area [35],

$$
F_{\mathrm{t}}=S_{\mathrm{c}}\left(\alpha A_{\text {intimate }}+\beta A_{\text {laid }}\right)
$$

considering that the frictional contributions of the intimate and laid parts of the contact can be added. $S_{\mathrm{c}}$ is the adhesive shear stress due to intermolecular forces as mentioned by Israelachvili [35,16], whereas $A_{\text {intimate }}$ and $A_{\text {laid }}$ are the apparent contact areas located within the external contour (dashed circles on Fig. 4 and 6). This implies that under certain circumstances, only a portion $\alpha$ and $\beta$ of, respectively, intimate and laid areas contributes to the friction. Thus $\alpha A_{\text {intimate }}$ and 

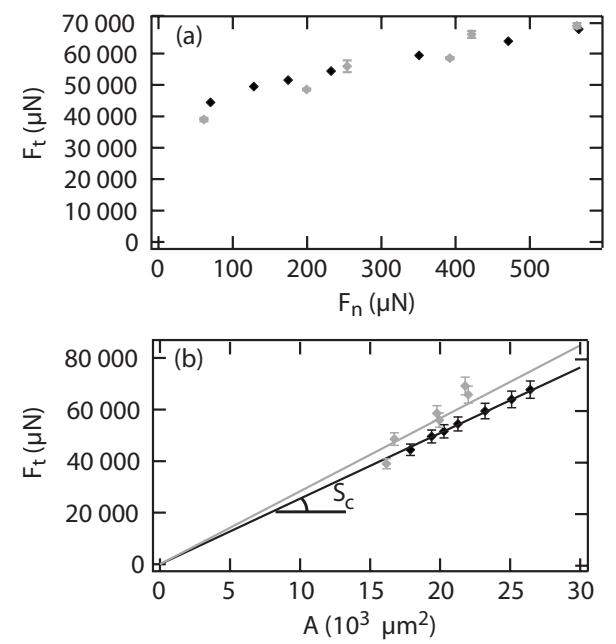

FIG. 5. $h=80 \mathrm{~nm}$ (a) Friction force $F_{\mathrm{t}}$ versus normal load $F_{\mathrm{n}}$ for $U=35 \mu \mathrm{m} / \mathrm{s}$ (black) and $U=350 \mu \mathrm{m} / \mathrm{s}$ (gray). (b) Friction force versus contact area $A$ for $U=35 \mu \mathrm{m} / \mathrm{s}$ (black) and $U=350 \mu \mathrm{m} / \mathrm{s}$ (gray).

$\beta A_{\text {laid }}$ are the friction contact areas. In order to validate the proposed friction law, the friction behavior of intimate, laid, and mixed contacts has been investigated.

\section{Intimate contact $(h=80 \mathrm{~nm})$ : Determination of $S_{c}$}

Description of sliding friction with time. Before the sliding begins at constant normal load, the static contact has a circular shape [see Fig. 4(a)i]. No pillar can be distinguished in the contact since the lens and the substrate are in intimate contact. At the onset of sliding, the substrate first sticks to the lens and then begins to slide [see Fig. 4(a)]. It can be seen in Fig. 4(a)iii that the contact front and back shrink and that its shape becomes oblong, whatever the sliding velocity is. The tangential force increases to a maximum when sliding begins. This corresponds to a static friction peak. Then it decreases to the kinetic value of friction and remains constant until the end of sliding (see Fig. 4). This observation has also been described by Barquins et al. [47]: first, adhesive forces keep the lens stuck on the substrate. The contact area is the same as at rest because the lens moves as one block, and the tangential force increases. As the contact pressure is lower on the contact edges, the lens unsticks more easily and microslidings occur at the front, but especially at the contact back. The latter slides forward, whereas the front slightly slides backward, which leads to an oblong shape of the contact.

Influence of normal load, contact area, and sliding velocity. Friction experiments have been carried out at two sliding velocities $U=35 \mu \mathrm{m} / \mathrm{s}$ and $U=350 \mu \mathrm{m} / \mathrm{s}$ for several normal loads. Figure 5(a) shows an increasing friction force $F_{t}$ with normal load $F_{n}$, independently of sliding velocity. However, as expected in the case of adhesive friction, the Coulomb's law $F_{t}=\mu F_{n}$, with $\mu$ the friction coefficient, is not obeyed here. The contact area $A_{\text {intimate }}$ can be measured and its evolution with normal load follows JKR law [13]. As a consequence, the friction force $F_{t}$ can be plotted versus the contact area $A_{\text {intimate }}$ in Fig. 5(b): it depends linearly on $A_{\text {intimate }}: F_{t}=S_{c} A_{\text {intimate }}$. The adhesive shear stress $S_{c}$ can be
TABLE I. Values of the shear stress $S_{\mathrm{c}}, \alpha$, and $\beta$.

\begin{tabular}{|c|c|c|c|c|c|}
\hline \multirow[b]{2}{*}{$\begin{array}{c}U \\
(\mu \mathrm{m} / \mathrm{s})\end{array}$} & \multicolumn{3}{|c|}{$h=80 \mathrm{~nm}$} & \multicolumn{2}{|c|}{$h=2900 \mathrm{~nm}$} \\
\hline & $\begin{array}{c}S_{\mathrm{c}} \\
(\mathrm{MPa})\end{array}$ & $\begin{array}{c}\alpha \\
(\%)\end{array}$ & $\beta$ & $\alpha$ & $\begin{array}{c}\beta \\
(\%)\end{array}$ \\
\hline 35 & $2.55 \pm 0.01$ & 100 & 0 & 0 & 26 \\
\hline 350 & $2.83 \pm 0.06$ & 100 & 0 & 0 & 25 \\
\hline
\end{tabular}

defined as the slope of the curve and its value is reported in Table I. The value is higher than usually found (e.g., Vorvolakos et al. [48]): this can be explained by the fact that both the sphere and the substrate are of the same material (PDMS), leading to commensurate surfaces, as observed for glassy polymers in Sivebaeck et al. [34]. It slightly increases with the sliding velocity. Such an increase with velocity is predicted by several theoretical models $[35,18,49,25,50]$ : they consider an elastic model where adhesive bindings are continuously stretched then broken. There is a regime where a rising extension of adhesive bindings is observed before they break, leading to an increasing shear stress with an increasing sliding velocity. A second explanation is the contribution of polymer chains friction, which can be seen as a viscous contribution [49].

\section{Laid contact $(h=2900 \mathrm{~nm})$ : Determination of $\beta$}

Description of sliding friction with time. Before the sliding begins, the static contact has a circular shape [see Fig. 6(a)i]. The pillars tops on which the lens lays can be clearly seen inside the contact. When the substrate begins to translate, the contact remains circular as shown in Fig. 6(a)ii.

We do not observe any static friction peak of the tangential force $F_{t}$, which increases directly to a kinetic friction value when the sliding begins, then remains constant until the end of sliding. The absence of static friction is due to a weaker adhesion of the lens on the substrate, as only the asperity tops are in contact with the lens.

During the sliding, we also observe within the contact that the pillars do not appear as dark disks as they do outside of
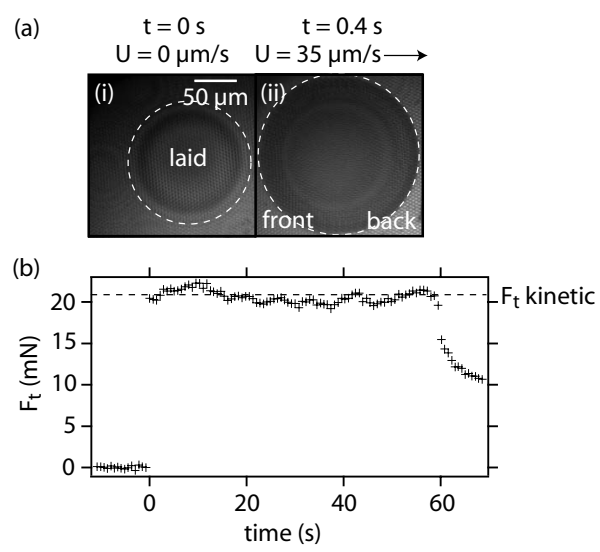

FIG. 6. $h=2900 \mathrm{~nm}$ : (a) Contact images when sliding begins: (i) before friction begins, and (ii) during friction. The arrow represents the sliding direction. (b) Friction force versus time for a constant normal load of $460 \mu \mathrm{N}$ and a sliding velocity of $35 \mu \mathrm{m} / \mathrm{s}$. 

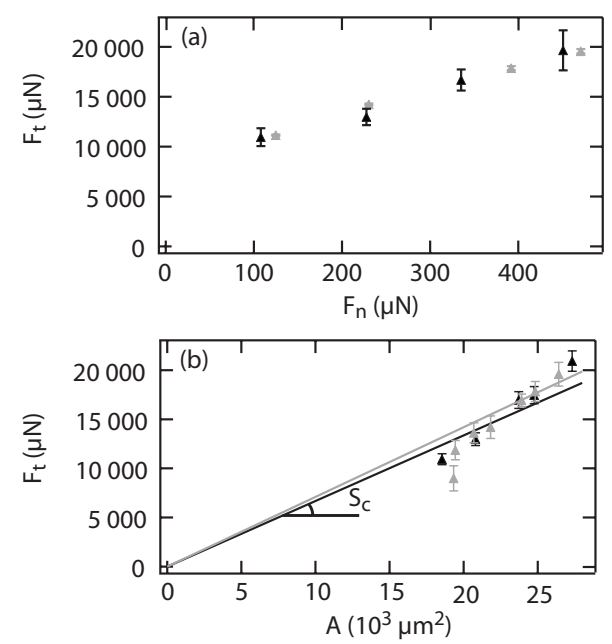

FIG. 7. $h=2900 \mathrm{~nm}$ (a) Friction force versus normal load for $U=35 \mu \mathrm{m} / \mathrm{s}$ (black) and $U=350 \mu \mathrm{m} / \mathrm{s}$ (gray). (b) Friction force versus contact area for $U=35 \mu \mathrm{m} / \mathrm{s}$ (black) and $U=350 \mu \mathrm{m} / \mathrm{s}$ (gray).

the contact. We clearly see streaks, which means that the pillars are bent. This can be explained by the following calculus: the elastic deformation energy for a pillar deformed on a length $r$ with a curvature radius $r$ is $\kappa r \sim 10^{-12} \mathrm{~J}$ with $\kappa$ $=E \pi r^{4} / 4$ the rigidity constant for a cylinder. This energy is of the same order of length as the friction force work on a pillar $F_{t} \lambda^{2} / 4 A_{\text {laid }} \sim 10^{-12} \mathrm{~J}$, as $F_{t} \sim 10 \mathrm{mN}$. The pillars bending also explains the fact that the dynamical contact [Fig. 6(a)ii] is greater than the static one [Fig. 6(a)i].

Influence of normal load, contact area and sliding velocity. Figure 7(a) shows an increasing friction force $F_{t}$ with normal load $F_{n}$, independently of sliding velocity. Once again we can see from Fig. 7(b) that the frictional response of the experimental system does not follow Coulomb's law. As the dynamical contact follows JKR law, we have plotted the friction force versus contact area and observed that the system obeys to an adhesive friction law, as previously. This time, however, $\alpha=0$ as there is no intimate contact. Since a laid contact can be seen as the discrete sum of many intimate contacts between the lens and the pillars tops, we propose to apply Eq. (3) with the value of $S_{c}$ found for $h=80 \mathrm{~nm}$, but with $\beta<100 \%$ because the friction contact area is lower than the area located with the circular contour,

$$
F_{t}=S_{c} \beta A_{\text {laid }}
$$

$\beta$ is thus expected to be close to the ratio of effective contact area over the apparent one, that is the pillar density $23 \%$. We find experimentally $\beta \sim 25 \%$. The fact that it is slightly higher than the geometric pillar density can be explained by the fact that the pillars are bent during sliding and the effective contact area is then higher. An estimation of the effective contact area from the contact pictures has been tried, but it has not been conclusive due to insufficient picture resolution.

\section{Mixed contact: $h=310 \mathrm{~nm}$ : Determination of $\alpha$ and $\beta$}

Description of sliding friction with time. Before the sliding begins, the contact has a circular shape and is entirely

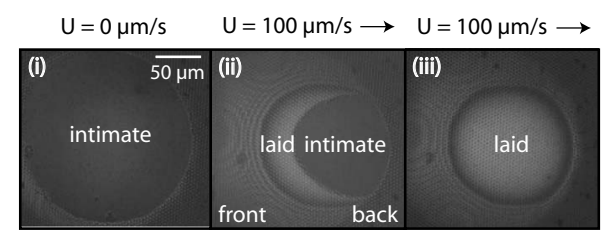

FIG. 8. $h=310 \mathrm{~nm}$ : Contact images when sliding begins: (i) before friction begins, (ii) and (iii) during friction. The arrow represents the sliding direction.

intimate [see Fig. 8i]. When friction begins, the contact is mixed. The front surfs on the pillars tops and is laid, whereas the back remains in an intimate state [see Fig. 8ii]. The contact pressure, which was uniform in a static state, is higher at the contact back when the contact slides [51].

Validity of the adhesive friction law. In this configuration, the coexistence of an intimate and a laid contact has allowed us to verify the friction law described in Eq. (3) for various sliding velocities. Assuming that the interfacial shear stress $S_{c}$ is known and taken as $2.55 \mathrm{MPa}$ at $U=35 \mu \mathrm{m} / \mathrm{s}$ and 2.85 $\mathrm{MPa}$ at 85 and $195 \mu \mathrm{m} / \mathrm{s}$, Fig. 9 presents the plot of $F_{t} / S_{c}$ - $\beta A_{\text {laid }}$ versus $A_{\text {intimate }}$, where $\beta$ has been chosen such that the plot passes through the origin: it can be seen that all experimental data form a straight line of slope $\alpha$. The small apparent variation of alpha with velocity remains unexplained. The value of $\alpha \sim 55 \%$ remains lower than $100 \%$. This result tends to demonstrate that even for an intimate contact with a pillars height of $310 \mathrm{~nm}$, only a fraction of the surface contributes to sliding. This can be due to a small gap between the lens and the ground of the substrate: the optical interval between a dark and a bright fringe is $273 \mathrm{~nm}$. Thus, if there is a gap of about $10 \mathrm{~nm}$ between the lens and the substrate between two pillars, it will unlikely be detected with the camera lateral resolution. Another explanation relies on the fact that only the effective surface occupied by the adhesive bindings should be considered [16]. In that case, the piece of information brought by $\alpha$ is the ratio between the effective surface of adhesive bindings between $h=310 \mathrm{~nm}$ and $h=80$ nm. $\alpha<100 \%$ means that the friction contact area is lower on higher pillars. This is predictable, as the elastic deformation is more important. The fact that the contact surface contributing to the friction is less than for an ideal entirely intimate contact is also reinforced by the low value of $\beta \sim 15 \%$ compared to the surface density occupied by one pillar, which would predict $\beta=23 \%$. We recall moreover that even for a bent pillar $(h=2900 \mathrm{~nm})$, the value found is about

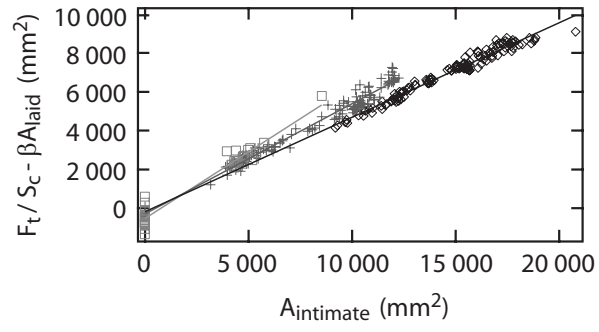

FIG. 9. Determination of $\alpha$ for three velocities $(h=310 \mathrm{~nm})$ : black diamond shaped: $U=35 \mu \mathrm{m} / \mathrm{s}$, dark gray cross: $U$ $=85 \mu \mathrm{m} / \mathrm{s}$, hell gray square: $U=195 \mu \mathrm{m} / \mathrm{s}$. 


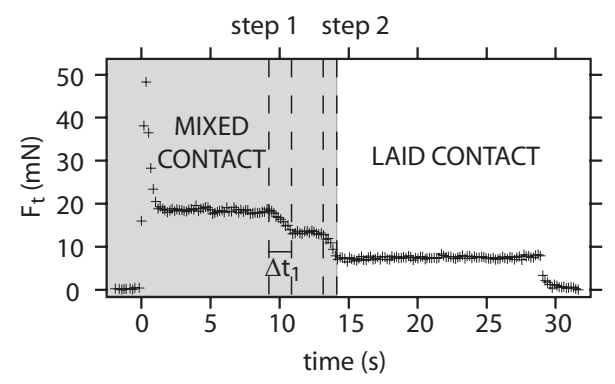

FIG. 10. Transition from a mixed (gray domain) to a laid contact in two steps.

$25 \%$. Finally, concerning the low value of $\beta$ found for $h$ $=310 \mathrm{~nm}$, a third argument can be proposed: the value of $23 \%$ fits for a cylindrical pillar with a flat disk of radius $1 \mu \mathrm{m}$ on its top, whereas the pillars of height $310 \mathrm{~nm}$ are spherical caps, thus when the lens lays on their tops, the contact surface is smaller.

\section{Conclusion}

In conclusion of this section, an adhesive friction law is found to govern the frictional behavior of PDMS micropatterned contacts: $F_{\mathrm{t}} \propto$ real contact area for intimate, laid and mixed contacts. The additivity of the contributions of two different kinds of contact (laid and intimate) is verified, and the presence of pillars lowers the fraction of contact contributing to sliding.

\section{Mixed to laid contact transition during sliding}

A transient phenomenon is observed during friction experiments on a $310 \mathrm{~nm}$ high pillared surface as shown in Fig. 10: a transition occurs from a mixed to a laid contact in two steps.

For sliding velocities lower than $35 \mu \mathrm{m} / \mathrm{s}$, the contact is always mixed: the front is laid and the back is intimate. For sliding velocities greater than $250 \mu \mathrm{m} / \mathrm{s}$, it is always laid. For sliding velocities ranging from $65 \mu \mathrm{m} / \mathrm{s}$ to $190 \mu \mathrm{m} / \mathrm{s}$, a transition from a mixed to a laid contact can be observed, while keeping the normal load constant and without tuning any experimental parameter. For this range of velocities, two experiments under similar values of velocity and normal load do not give rise to exactly the same observations: the transition can be observed or not. This can be explained by the high sensitivity of this transition to experimental conditions (pillars defects or presence of dust for example). However, for each velocity between 35 and $190 \mu \mathrm{m} / \mathrm{s}$, ten experiments have been made: Fig. 11 presents a map of coexistence of these contact states as a function of the sliding velocity and the corresponding measured friction force is also reported for a constant normal load of $320 \mu \mathrm{N}$. This figure shows that the transition mixed $\rightarrow$ laid occurs more often when the sliding velocity increases.

A careful examination of the mixed/laid contact transition shows that it occurs in two steps. First, the intimate part reduces, and second it disappears. Thus, two transition velocities can be defined: on the one hand, $u_{1}$, defined with respect to the lens frame, corresponds to the first step and is

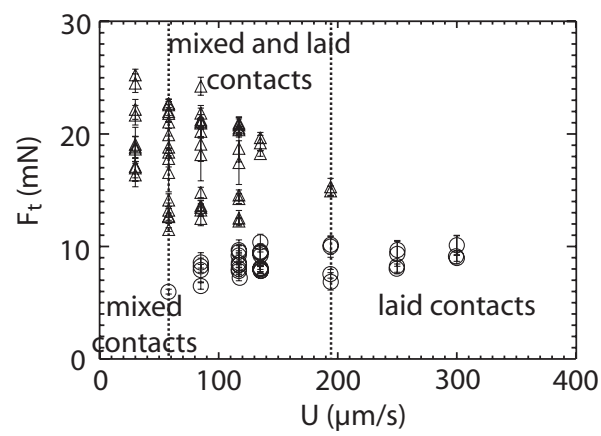

FIG. 11. Map of occurrences of the mixed $\rightarrow$ laid transition: friction force versus sliding velocity $U$ for a normal load $F_{n}$ $=320 \mu \mathrm{N}$. Mixed (black triangles) and laid (black circles) contacts are observed.

about $25 \pm 15 \mu \mathrm{m} / \mathrm{s}$. It does not depend on sliding velocity $U$. Moreover, $u \times \Delta t_{1}$ corresponds to the distance covered by the intimate contact during the transition. On the other hand, $u_{2}$, corresponding to the second step, increases with sliding velocity $U$ as reported in Table II. During the two transition steps, the friction force and the intimate area decrease whereas the tangential stress remains constant at about 1.5 $\mathrm{MPa}$ (respectively, $1 \mathrm{MPa}$ ) for the first step (respectively for the second step) (values for $U=115 \mu \mathrm{m} / \mathrm{s}$ ).

The existence of this transition can be correlated with the competition taking place between the sliding velocity, which tends to lift the lens over the substrate, and the spreading velocity of the lens on the pillars which favors the existence of an intimate state. Spreading experiments have been carried out [52] and have shown that the spreading of the intimate state in static conditions originates from nucleationgrowth phenomena and the spreading velocity has been estimated at about $100 \mu \mathrm{m} / \mathrm{s}$ [53-58]. Therefore, this competition can explain the sliding velocity dependance of the transition:

(i) at low sliding velocities $U \ll 100 \mu \mathrm{m} / \mathrm{s}$, a mixed contact is established between the lens and the substrate,

(ii) at intermediate sliding velocities $U \sim 100 \mu \mathrm{m} / \mathrm{s}$, the competition takes place and the transition between mixed and laid contacts occurs,

(iii) at high sliding velocities $U>100 \mu \mathrm{m} / \mathrm{s}$, laid contacts predominate.

The transition from a laid to a mixed contact has not been observed, or it has been clearly induced by the passage of a dust.

TABLE II. Second step transition velocity with respect to the lens for different sliding velocities $U$.

\begin{tabular}{|c|c|}
\hline $\begin{array}{l}U \\
(\mu \mathrm{m} / \mathrm{s})\end{array}$ & $\begin{array}{c}\text { Transition velocity } \\
(\mu \mathrm{m} / \mathrm{s})\end{array}$ \\
\hline 60 & $62 \pm 6$ \\
\hline 120 & $104 \pm 16$ \\
\hline 135 & $101 \pm 10$ \\
\hline 160 & $137 \pm 40$ \\
\hline
\end{tabular}




\section{CONCLUSION}

A home made tribometer similar to a macroscopic AFM has been used to investigate the influence of pillars height, normal load, and sliding velocity on the friction of a flat PDMS lens on a textured PDMS substrate, by the way of friction force measurements and contact observations.

The contact observations show the existence of two types of contacts, intimate on small pillars and laid on high ones. Moreover, for pillars of intermediate height $310 \mathrm{~nm}$, a mixed contact, where the back is intimate and the front laid, has been observed. The existence of intimate and laid contacts is explained by the competition between PDMS/PDMS adhesion and elastic deformation resulting from the creation of an intimate contact area.

The frictional response of the system does not follow a classical Coulomb law in the range of chosen experimental conditions. Adhesion plays a dominant role, and an adhesive friction law, similar to the one found for molecularly smooth surfaces [35] should be considered: $F_{t}=S_{c}\left(\alpha A_{\text {intimate }}\right.$ $\left.+\beta A_{\text {laid }}\right) . \alpha$ (respectively, $\beta$ ) represents the percentage of measured area $A_{\text {intimate }}$ (respectively, $A_{\text {laid }}$ ) which contributes to the friction, and are close to the values computed from geometrical considerations of the pillars. The shear stress due to intermolecular forces $S_{c}$ increases slightly with sliding velocity $U$.

Furthermore, we show that a transition from an intimate to a laid contact occurs for $h=310 \mathrm{~nm}$ when sliding velocity increases. This transition originates from a competition between the spreading velocity of an intimate contact in a laid contact, which is close to $100 \mu \mathrm{m} / \mathrm{s}$, and the sliding velocity $U$.
[1] W. Ehrfeld and D. Münchmeyer, Nucl. Instrum. Methods Phys. Res. A 303, 523 (1991).

[2] M. Harmeming, W. Bacher, P. Bley, A. El-Kholi, H. Kalb, B. Kowanz, W. Menz, A. Michel, and J. Mohr, Molding of ThreeDimensional Microstructures by the Liga Process, Proceedings of the IEEE MEMS Workshop (IEEE Computer Society Press, Travemünde, 1992), pp. 202-207.

[3] S. Megert, A. Labeque, Liu. Zewen, H. Dexpert, R. Comès, F. Rousseaux, M. F. Ravet, H. Launois, S. Ballandras, W. Daniau, S. Basrour, M. Rouillay, P. Blind, and D. Hauden, Tribol. Lett. 4, 269 (1994).

[4] J. Mohr, W. Ehrfeld, D. Münchmeyer, and A. Stutz, Macromol. Symp. 97, 231 (1989).

[5] P. Joseph, C. Cottin-Bizonne, J.-M. Benoit, C. Ybert, C. Journet, P. Tabeling, and L. Bocquet, Phys. Rev. Lett. 97, 156104 (2006).

[6] D. Quéré and M. Reyssat, Philos. Trans. R. Soc. London, Ser. A 366, 1539 (2008).

[7] Y. Tian, N. Pesika, H. Zheng, K. Rosenberg, B. Zhao, P. McGuiggan, K. Autumn, and J. Israelachvili, Proc. Natl. Acad. Sci. U.S.A. 103, 19320 (2006).

[8] J. N. Israelachvili, Nature (London) 435, 893 (2005).

[9] T. Baumberger and C. Caroli, Adv. Phys. 55, 279 (2006).

[10] B. N. J. Persson, Surf. Sci. Rep. 61, 201 (2006).

[11] C. Yang, B. N. J. Persson, J. Israelachvili, and K. Rosenberg, EPL 84, 46004 (2008).

[12] K. N. G. Fuller and D. Tabor, Proc. R. Soc. London, Ser. A 345, 327 (1975).

[13] K. L. Johnson, K. Kendall, and A. S. Roberts, Proc. R. Soc. London, Ser. A 324, 301 (1971).

[14] B. N. J. Persson and E. Tossati, J. Chem. Phys. 115, 5597 (2001).

[15] A. G. Peressadko, N. Hosoda, and B. N. J. Persson, Phys. Rev. Lett. 95, 124301 (2005).

[16] J. N. Israelachvili, S. Giasson, T. Kuhl, C. Drummond, A. Berman, G. Luengo, J.-M. Pan, M. Heuberger, W. Ducker, and N. Alcantar, Tribol. Ser. 38, 3 (2000).

[17] E. Verneuil, B. Ladoux, A. Buguin, and P. Silberzan, J. Adhes. 83, 449 (2007).
[18] A. Schallamach, Wear 6, 375 (1963).

[19] K. A. Grosch, Proc. R. Soc. London, Ser. A 274, 21 (1963).

[20] Yu. B. Chernyak and A. I. Leonov, Wear 108, 105 (1986).

[21] A. R. Savkoor, Wear 8, 222 (1965).

[22] K. C. Ludema and D. Tabor, Wear 9, 329 (1966).

[23] S. Sills, K. Vorvolakos, M. K. Chaudhury, and R. M. Overney, Molecular origins of elastomeric friction, Nanotribology: Friction and Wear on the Atomic Scale, (Springer-Verlag, Berlin, 2007), pp. 659-676.

[24] S. Sills, T. Gray, and R. M. Overney, J. Chem. Phys. 123, 134902 (2005).

[25] C. Drummond, J. Israelachvili, and P. Richetti, Phys. Rev. E 67, 066110 (2003).

[26] J. Cayer-Barrioz, D. Mazuyer, A. Tonck, and E. Yamaguchi, Langmuir 25, 10802 (2009).

[27] P.-G. De Gennes, Scaling concepts in Polymer Physics (Cornell University Press, Ithaca, New York, 1985).

[28] A. Schallamach, Proc. Phys. Soc. London, Sect. B 65, 657 (1952).

[29] Y. Okamoto, K. Nishio, J. Sugiura, M. Hirano, and T. Nitta, J. Phys.: Conf. Ser 89, 012011 (2007).

[30] B. He, W. Chen, and Q. Jane Wang, Tribol. Lett. 31, 187 (2008)

[31] B. J. Briscoe and D. Tabor, Wear 34, 29 (1975).

[32] A. M. Homola, J. N. Israelachvili, P. M. McGuiggan, and M. L. Gee, Wear 136, 65 (1990).

[33] P. G. Whitten and H. R. Brown, Phys. Rev. E 76, 026101 (2007).

[34] I. M. Sivebaeck, V. N. Samoilov, and B. N. J. Persson, Eur. Phys. J. E 27, 37 (2008).

[35] J. N. Israelachvili, N. Maeda, K. J. Rosenberg, and M. Akbulut, J. Mater. Res. 20, 1952 (2005).

[36] F. Deleau, D. Mazuyer, and A. Koenen, Tribol. Int. 42, 149 (2009)

[37] L. Bureau, T. Baumberger, and C. Caroli, Eur. Phys. J. E 19, 163 (2006).

[38] B. N. J. Persson, Wear 264, 746 (2008).

[39] A. Chateauminois and C. Fretigny, Eur. Phys. J. E 27, 221 (2008). 
[40] B. Zappone, K. J. Rosenberg, and J. Israelachvili, Tribol. Lett. 26, 191 (2007).

[41] L. Kogut and I. Etsion, Tribol. Lett. 126, 34 (2004).

[42] F. Heslot, T. Baumberger, B. Perrin, B. Caroli, and C. Caroli, Phys. Rev. E 49, 4973 (1994).

[43] A. Schallamach, Wear 17, 301 (1971).

[44] J. P. Cleveland, S. Manne, D. Bocek, and P. K. Hansma, Rev. Sci. Instrum. 64, 403 (1993).

[45] R. D. Blevins, Formulas for Natural Frequency and Mode Shape (Van Nostrand Reinhold, New York, 1979).

[46] Polymer Data Handbook, edited by J. E. Mark (Oxford University Press, New York, 1999).

[47] M. Barquins and R. Courtel, Wear 32, 133 (1975).

[48] K. Vorvolakos and M. K. Chaudhury, Langmuir 19, 6778 (2003).

[49] F. Brochard-Wyart and P.-G. De Gennes, Eur. Phys. J. E 23,
439 (2007).

[50] B. N. J. Persson and A. I. Volokitin, Eur. Phys. J. E 21, 69 (2006).

[51] J. Scheibert, Ph.D. thesis, Université Paris 6, 2007.

[52] F. Wu-Bavouzet, Ph.D. thesis, Université Paris 6, 2008.

[53] H. R. Brown, Science 263, 1411 (1994).

[54] A. Casoli, M. Brendlé, J. Schultz, P. Auroy, and G. Reiter, Langmuir 17, 388 (2001).

[55] J. A. Greenwood and J. B. P. Williamson, Proc. R. Soc. London, Ser. A 295, 300 (1966).

[56] C. Ishino, K. Okumura, and D. Quéré, EPL 68, 419 (2004).

[57] A. Martin, J. Clain, A. Buguin, and F. Brochard-Wyart, Phys. Rev. E 65, 031605 (2002).

[58] F. Michel and M. E. R. Shanahan, C. R. Acad. Sci. Paris 310, 17 (1990). 